\title{
Probing key organic substances driving new particle growth initiated by iodine nucleation in coastal atmosphere
}

Yibei Wan et al.

Correspondence to: Huan Yu (yuhuan@cug.edu.cn)

The copyright of individual parts of the supplement might differ from the CC BY 4.0 License. 


\section{Text:}

ESI-FT-ICR MS operation conditions

\section{Figures:}

Figure S1: Figure S1. (a) The observation site, indicated as a red star, in an aerial photograph. Photo source: Baidu Map. (b) 72-hour air mass back trajectories ending at $100 \mathrm{~m}$ above ground level at the observation site computed by HYSPLIT model during the I-NPF events from May 8 to 10, 2018.

Figure S2. (a), (c) Number concentration of 2-7 nm particles $\left(N_{2-7}\right)$, tidal height and solar radiation intensity during the Iodine-initiated NPF (I-NPF) days from May 9 to 11 and the continental regional NPF (C-NPF) days from February 11 to 13. Particle number size distribution and 10-56 nm particle mass concentration during (b) I-NPF days from May 9 to 11, (d) C-NPF days from February 11 to 13 and (e) non-NPF days from April 16 to 18 .

Figure S3. Reconstructed mass spectra of the 7 elemental groups in ESI-and ESI+ modes for the four size bins.

Figure S4: DBE vs. C atom number diagrams of all the $\mathrm{CHON}$ and $\mathrm{CHO}$ formulas detected in 10-18 nm particles in ESI+ mode. (a) (b) $+\mathrm{H}$ adducts, (c) (d) $+\mathrm{Na}$ adducts. The color bar denotes the $\mathrm{O}$ number in the formulas. The size of the circles reflects the relative intensities of molecular formulas on a logarithmic scale.

Figure S5: Relative intensities of subgroups according to $\mathrm{O}$ atom number in $\mathrm{CHON}$, $\mathrm{CHO}, \mathrm{CHONI}$ and $\mathrm{CHOI}$ formulas in the four size bins in ESI+ (in red) and ESI- (in blue). The intensity of the most abundant subgroup is defined as 1 and those of other subgroups are normalized by it. The relative intensities of non-iodinated OC formulas (iodinated OC formulas) are plotted in the region above (below) zero line.

Figure S6: $\mathrm{O}$ atom number of $v s . \mathrm{N}$ atom number of $\mathrm{C}_{10} \mathrm{H}_{\mathrm{x}} \mathrm{O}_{\mathrm{y}} \mathrm{N}_{\mathrm{z}}$ compounds detected in 180-560 nm particles (a) and $\mathrm{C}_{18} \mathrm{H}_{\mathrm{x}} \mathrm{O}_{\mathrm{y}} \mathrm{N}_{\mathrm{z}}$ compounds detected in 10-18 nm particles in ESI- mode (b).

Figure S7: Simplified reaction scheme showing the formation of oxygenated and nitrated $\mathrm{CHO}$ and $\mathrm{CHON}$ compounds from $\alpha$-linolenic acid oxidation in the atmosphere. 
Figure S8: Simplified reaction scheme showing the formation of oxygenated $\mathrm{CHO}$ compounds from unsaturated $\mathrm{C}_{28} \mathrm{FA}\left(\mathrm{C}_{28} \mathrm{H}_{52} \mathrm{O}_{2}\right)$ oxidation in the atmosphere.

Figure S9: Simplified reaction scheme showing the formation of oxygenated CHON compounds containing a $-\mathrm{NH}_{2}$ group from unsaturated $\mathrm{C}_{18}$ amino alcohol $\left(\mathrm{C}_{18} \mathrm{H}_{37} \mathrm{NO}_{4}\right)$ oxidation in the atmosphere.

\section{Tables:}

Table S1. Predicted saturation concentration $\left(\mathrm{C}^{*}\right)$ range of most abundant $\mathrm{CHON}$ and $\mathrm{CHO}$ formulas, as well as their possible precursors. 


\section{ESI-FT-ICR MS operation conditions}

A syringe pump infused the sample extract continuously into the ESI unit with a flow rate of $180 \mu \mathrm{L}$ h-1. The ESI source conditions were as follows: the nebulizer gas pressure was 1 bar; the dry gas $\left(\mathrm{N}_{2}\right)$ pressure was 4 bar and its temperature was $200{ }^{\circ} \mathrm{C}$; the capillary voltage was $4.5 \mathrm{kV}$. The ion accumulation time in the argon-filled hexapole collision pool with $1.5 \mathrm{~V}$ of direct current voltage and 1400 Vp-p of radio frequency (RF) amplitude was $0.05 \mathrm{~s}$, followed by transport ions through a hexapole ion guide to the ICR cell for $0.7 \mathrm{~ms} .4 \mathrm{M}$ words of data were recorded over the mass range of 150-1000 for each run. A total of 128 scans were collected to enhance the signal/noise $(\mathrm{S} / \mathrm{N})$ ratio and dynamic range. 
(a)

(b)

NOAA HYSPLIT MODEL GDAS Meteorological Data
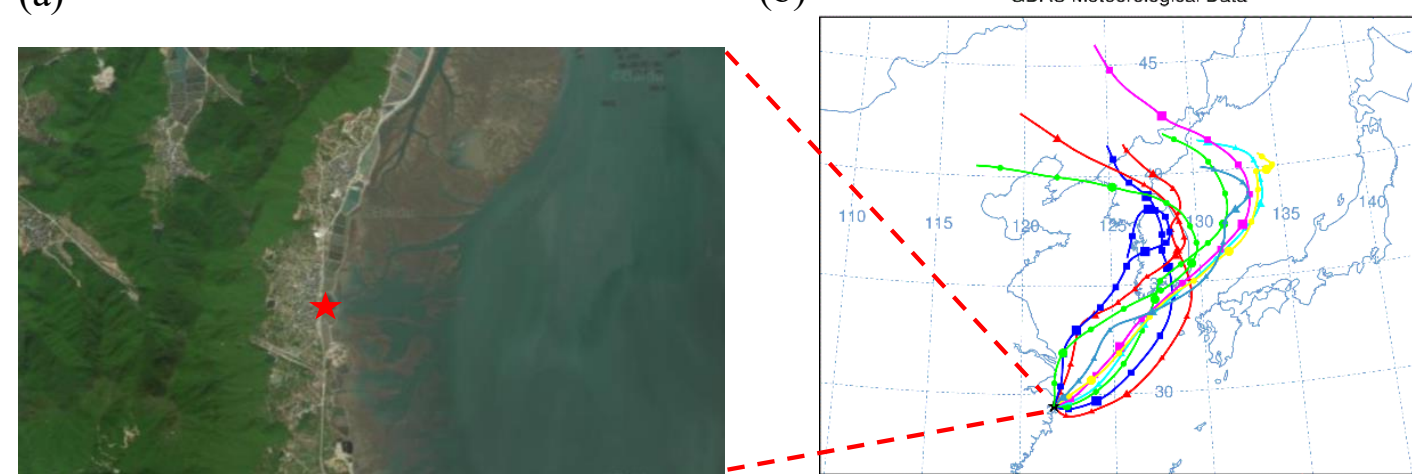

Figure S1. (a) The observation site, indicated as a red star, in an aerial photograph.

Photo source: Baidu Map. (b) 72-hour air mass back trajectories ending at 100

m above ground level at the observation site computed by HYSPLIT model

during the I-NPF events from My 8 to 10, 2018. 

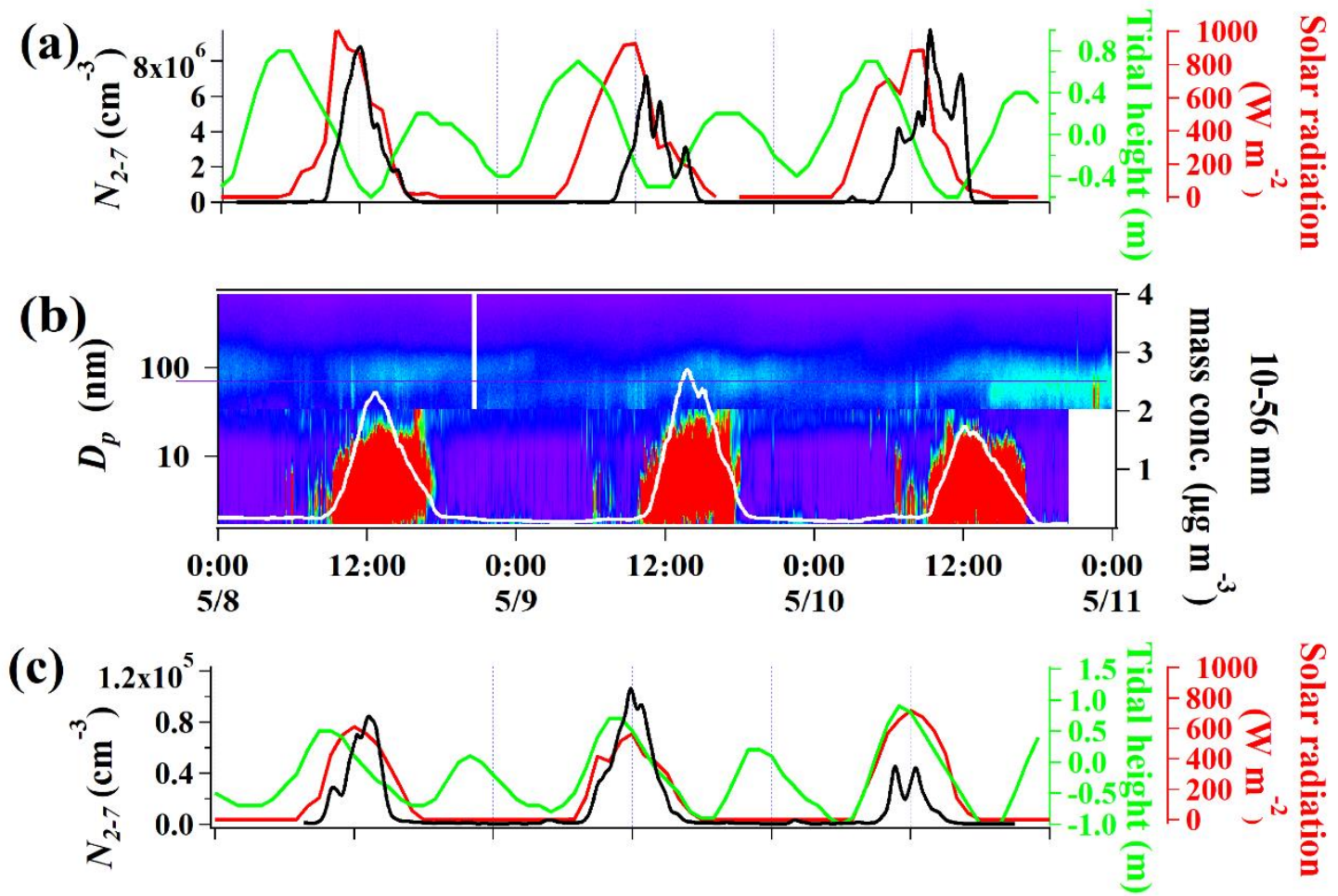

(d)

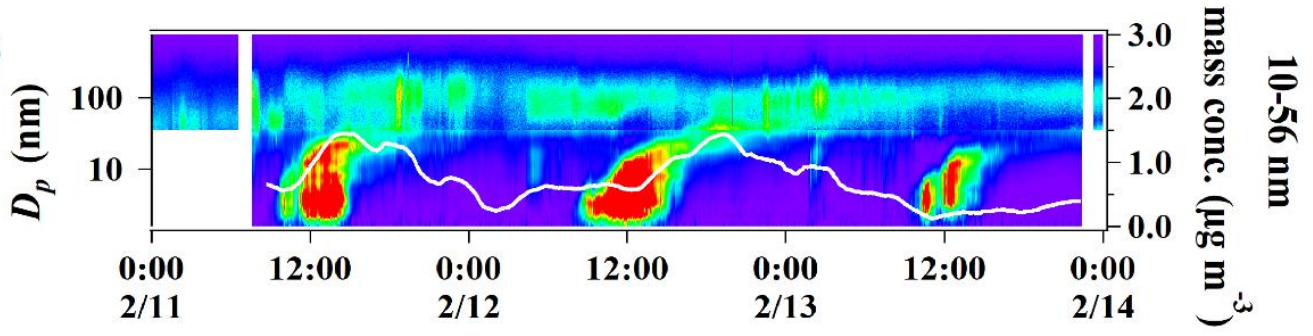

(e)
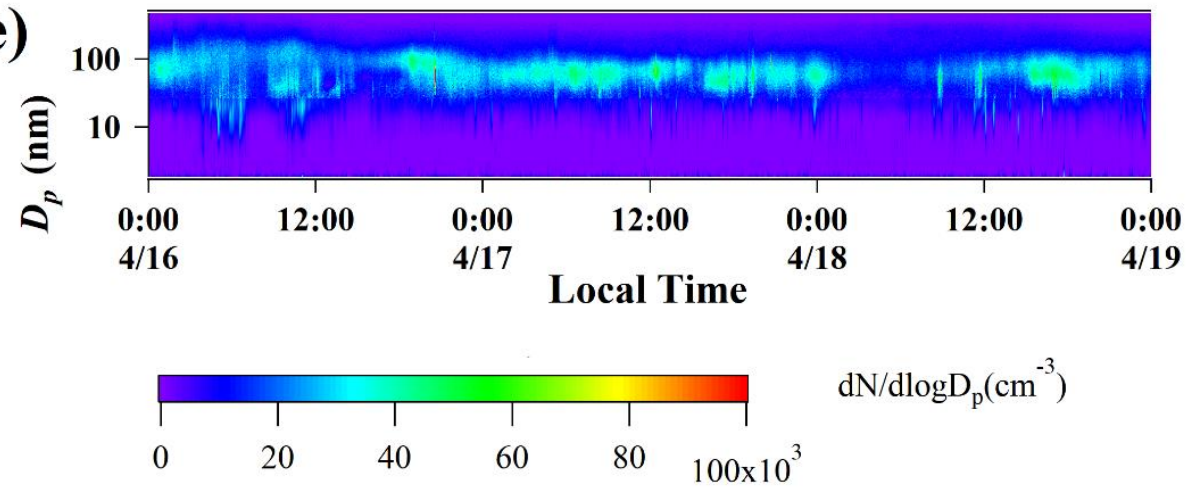
Figure S2. (a), (c) Number concentration of 2-7 nm particles $\left(N_{2-7}\right)$, tidal height and solar radiation intensity during the Iodine-initiated NPF (I-NPF) days from May 9 to 11 and the continental regional NPF (C-NPF) days from February 11 to 13.

Particle number size distribution and 10-56 nm particle mass concentration during (b) I-NPF days from May 9 to 11, (d) C-NPF days from February 11 to 13 and (e) non-NPF days from April 16 to 18. 

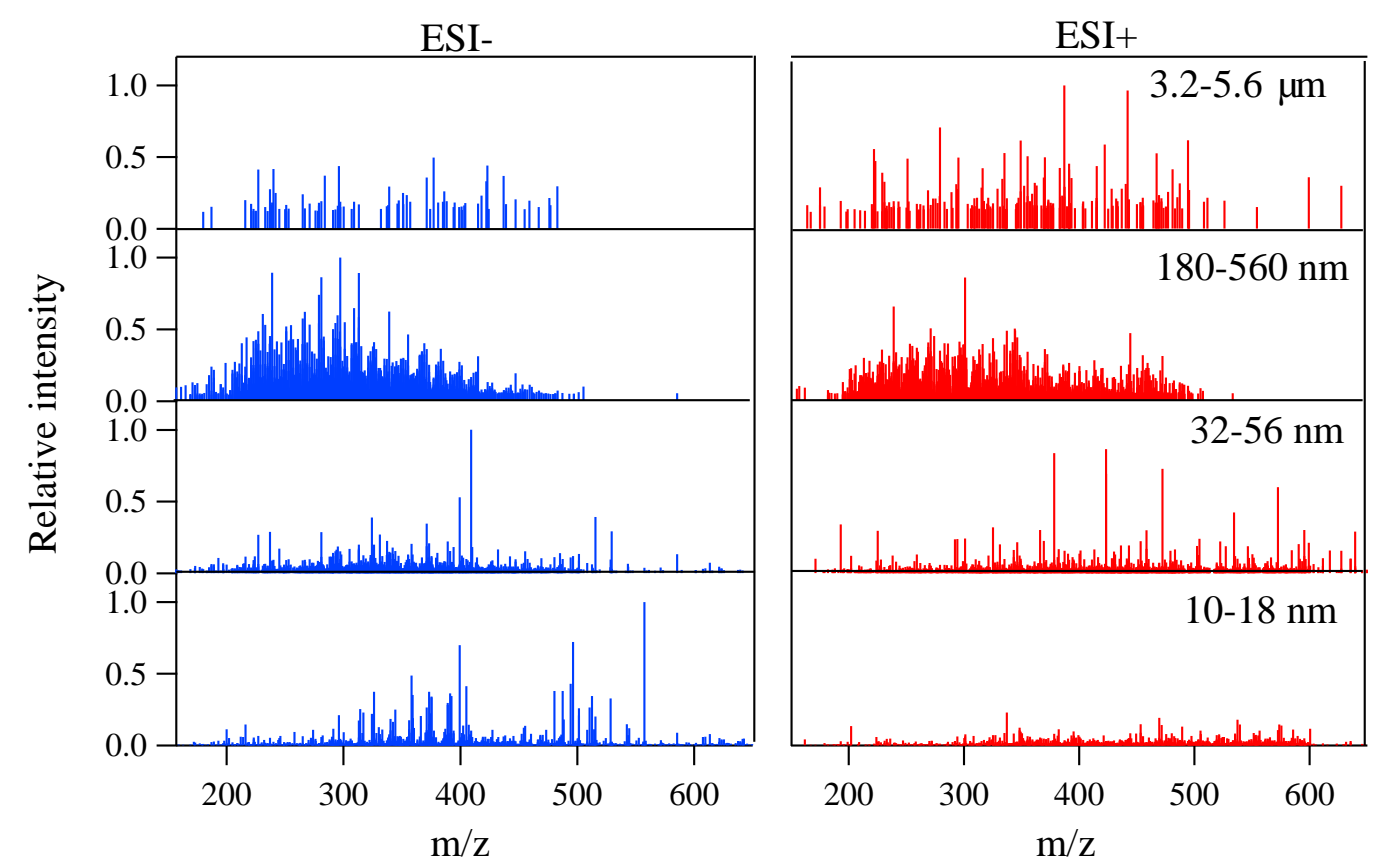

Figure S3. Reconstructed mass spectra of the 7 elemental groups in ESI- (left panels)

and ESI+ (right panels) modes for the four size bins. The signals are normalized against the intensity of the most abundant molecular ions in a size bin. 

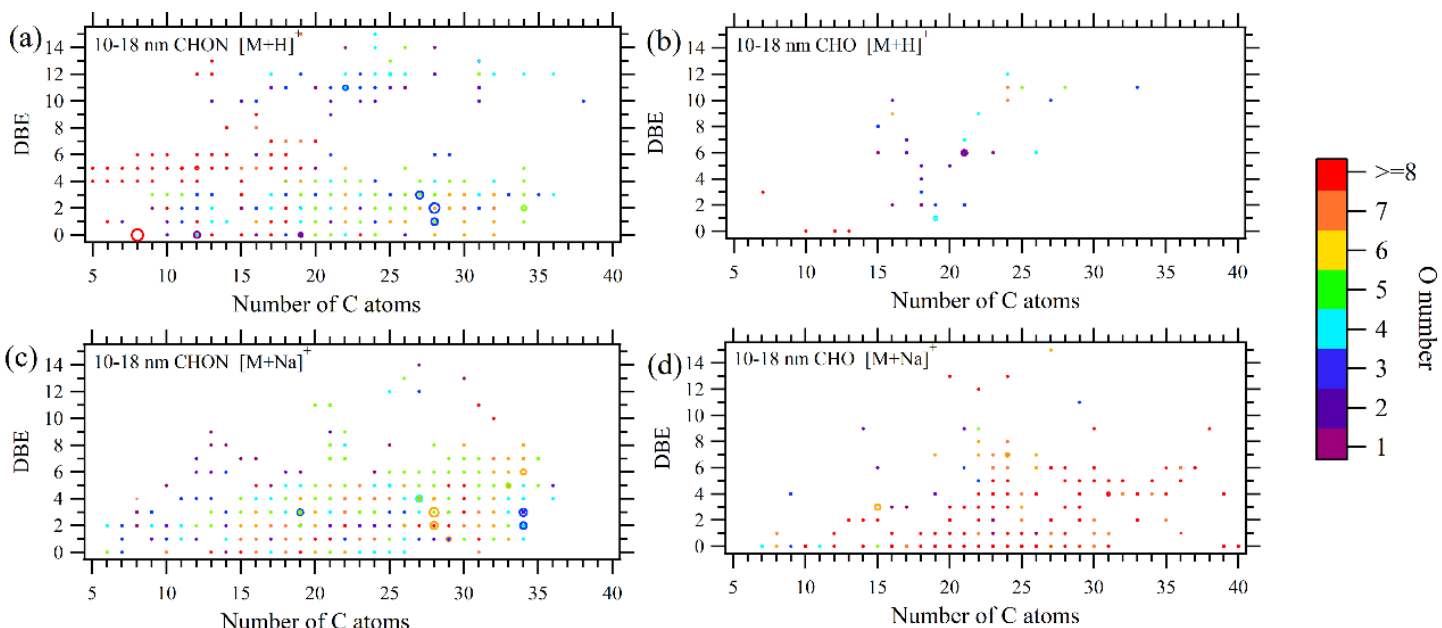

Figure S4. DBE vs. C atom number diagrams of all $\mathrm{CHON}$ and $\mathrm{CHO}$ formulas detected in 10-18 nm particles in ESI+ mode. (a) (b) $[\mathrm{M}+\mathrm{H}]^{+}$adducts, (c) (d) $[\mathrm{M}+\mathrm{Na}]^{+}$adducts. The color bar denotes $\mathrm{O}$ number in the formulas. The size of the circles reflects the relative intensities of molecular formulas on a logarithmic scale. 


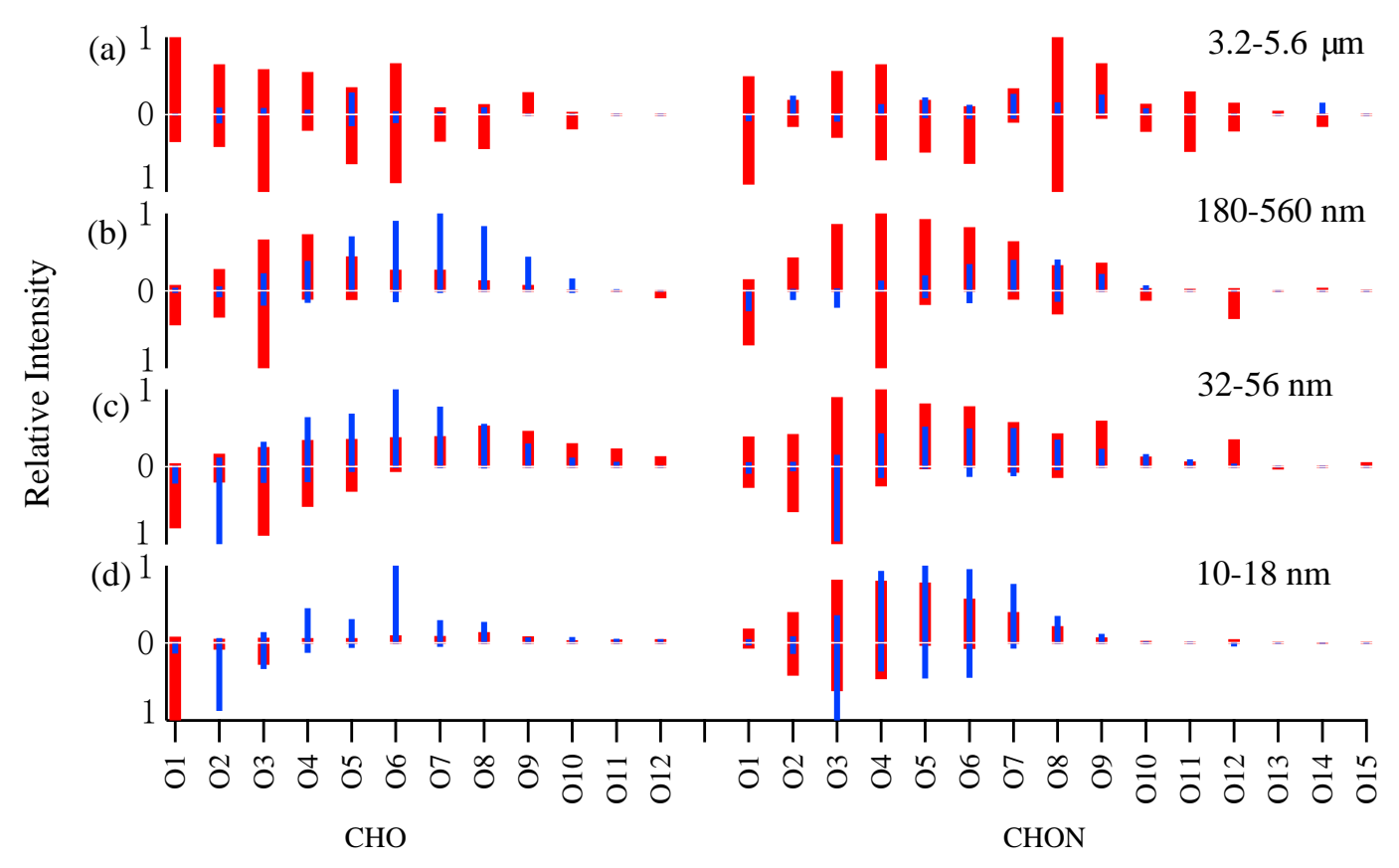

Figure S5. Relative intensities of subgroups according to $\mathrm{O}$ atom number in $\mathrm{CHON}$, $\mathrm{CHO}, \mathrm{CHONI}$ and $\mathrm{CHOI}$ formulas in the four size bins in ESI+ (in red) and ESI- (in blue). The intensity of the most abundant subgroup in a size bin is defined as 1 and those of other subgroups are normalized by it. The relative intensities of non-iodinated OC formulas (iodinated OC formulas) are plotted in the region above (below) zero line. 

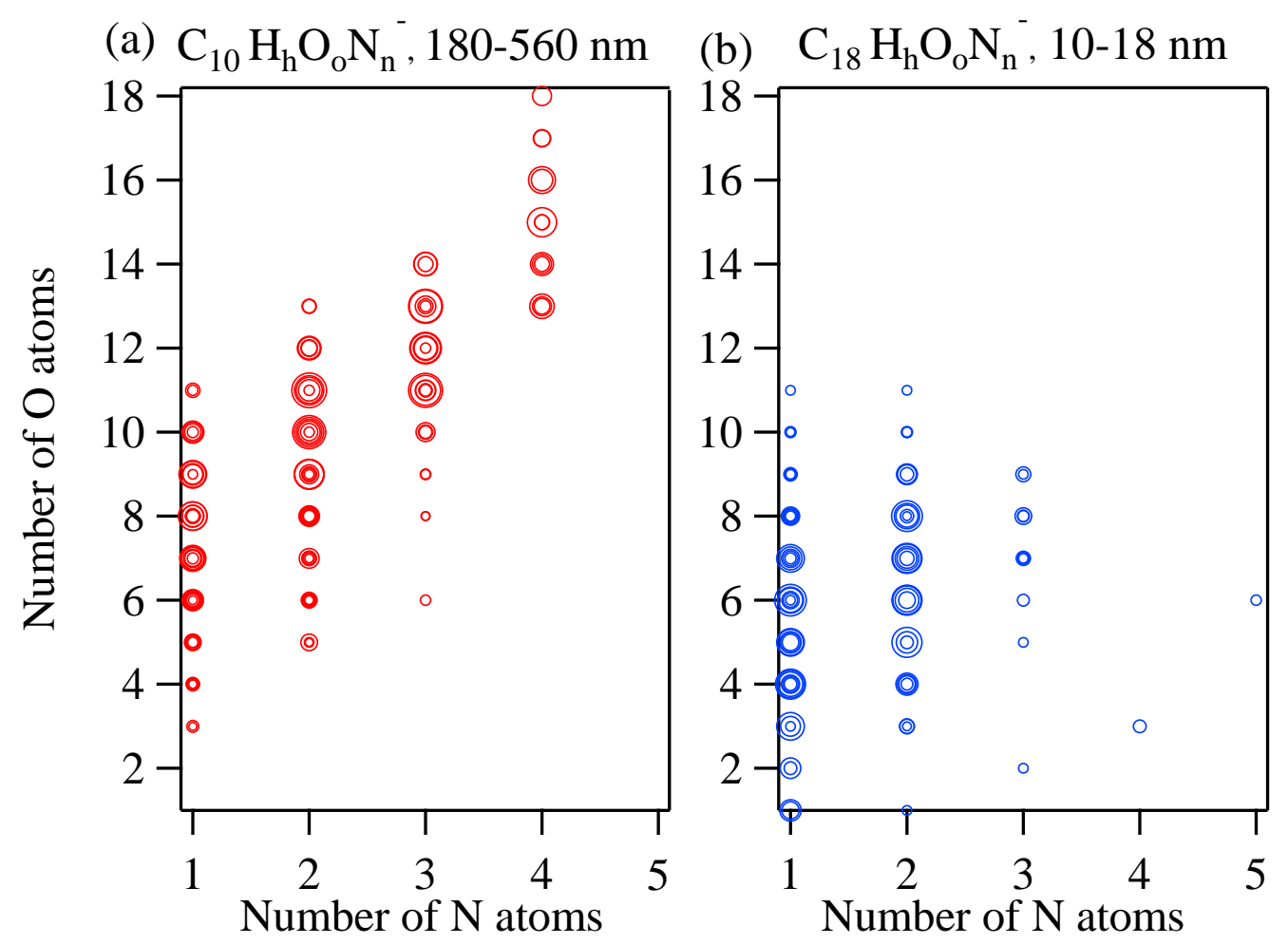

Figure S6. $\mathrm{O}$ atom number of $v s . \mathrm{N}$ atom number of $\mathrm{C}_{10} \mathrm{H}_{\mathrm{h}} \mathrm{O}_{0} \mathrm{~N}_{\mathrm{n}}{ }^{-}$compounds detected in 180-560 nm particles (a) and $\mathrm{C}_{18} \mathrm{H}_{\mathrm{h}} \mathrm{O}_{\mathrm{o}} \mathrm{N}_{\mathrm{n}^{-}}$compounds detected in 10-18 nm particles in ESI- mode (b). 


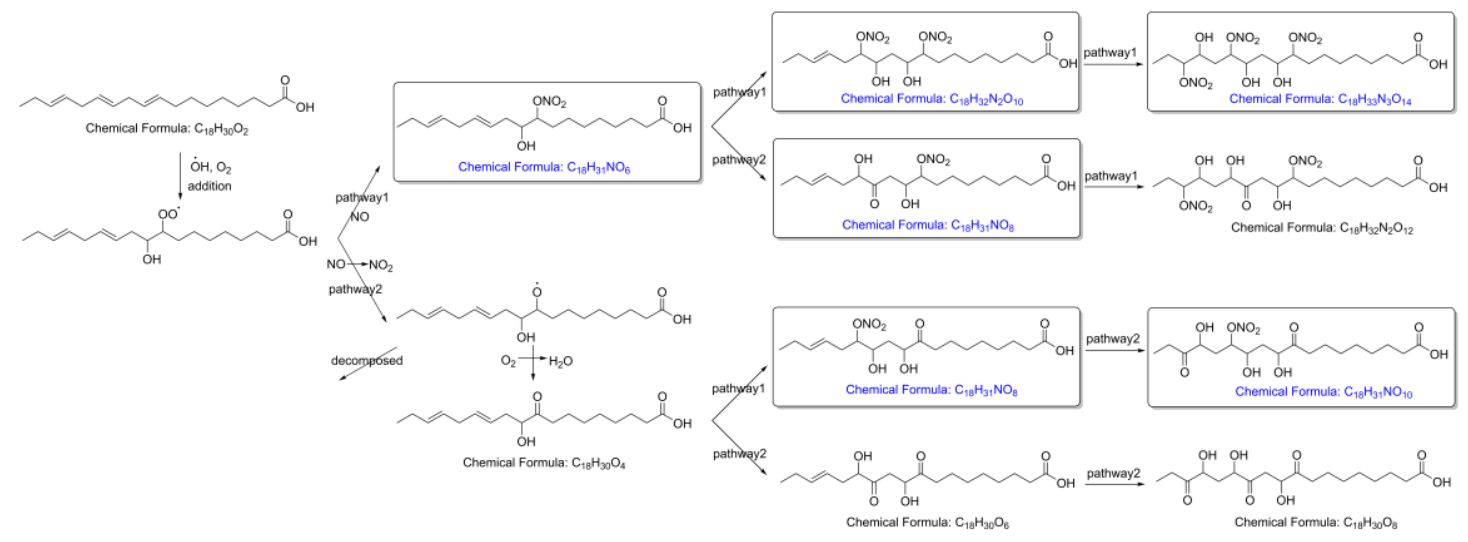

Figure S7. Simplified reaction scheme of the formation of oxygenated and nitrated CHO and CHON compounds from $\alpha$-linolenic acid $\left(\mathrm{C}_{18} \mathrm{H}_{30} \mathrm{O}_{2}\right)$ oxidation in the atmosphere. One representative structure is shown for each chemical formula. Chemical formulas in the boxes are found in the formula list detected in $10-18 \mathrm{~nm}$ particles. Pathway 1: $\mathrm{OH}$ and $\mathrm{O}_{2}$ addition followed by reaction with $\mathrm{NO}$ to form a $\mathrm{ONO}_{2}$ group; pathway 2: $\mathrm{OH}$ and $\mathrm{O}_{2}$ addition followed by reaction with $\mathrm{NO}$ to form an alkoxy radical that further reacts with $\mathrm{O}_{2}$ to form a $-\mathrm{C}=\mathrm{O}$ group. 


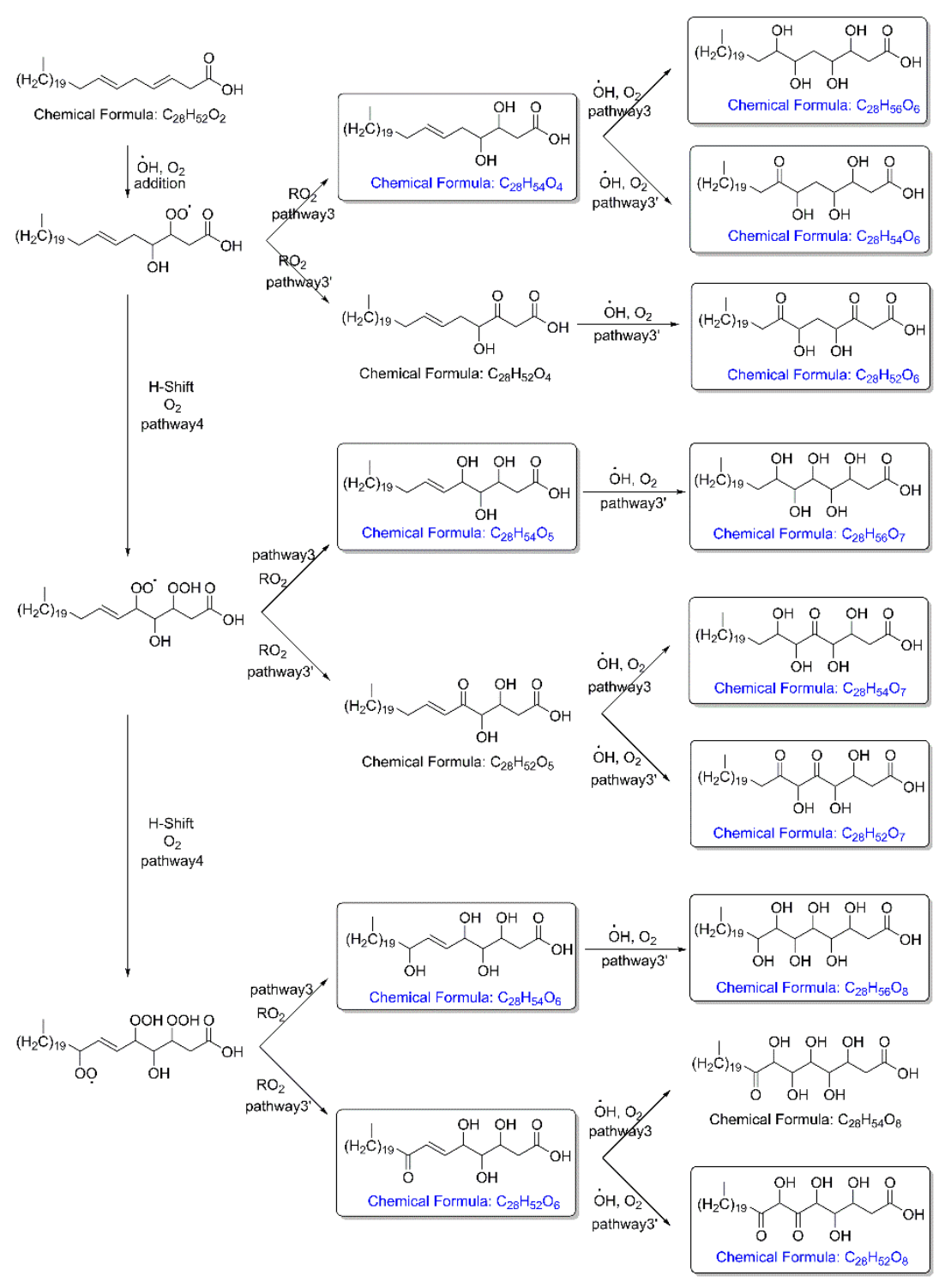

Figure S8. Simplified reaction scheme of the formation of oxygenated CHO compounds from unsaturated $\mathrm{C}_{28}$ FA $\left(\mathrm{C}_{28} \mathrm{H}_{52} \mathrm{O}_{2}\right)$ oxidation in the atmosphere. One representative structure is shown for each chemical formula. Chemical formulas in the boxes are found in the formula list detected in 10-18 nm particles. Pathway 3: $\mathrm{OH}$ and $\mathrm{O}_{2}$ addition followed by reaction with $\mathrm{RO}_{2}$ to form a $-\mathrm{OH}$ or a $-\mathrm{C}=\mathrm{O}$ group; Pathway 4 : successive intermolecular $\mathrm{H}$-shift/ $\mathrm{O}_{2}$ addition (autoxidation) to form $\mathrm{RO}_{2}$ radicals with $-\mathrm{OOH}$ group. $-\mathrm{OOH}$ group is not stable and decomposed to $-\mathrm{OH}$. 


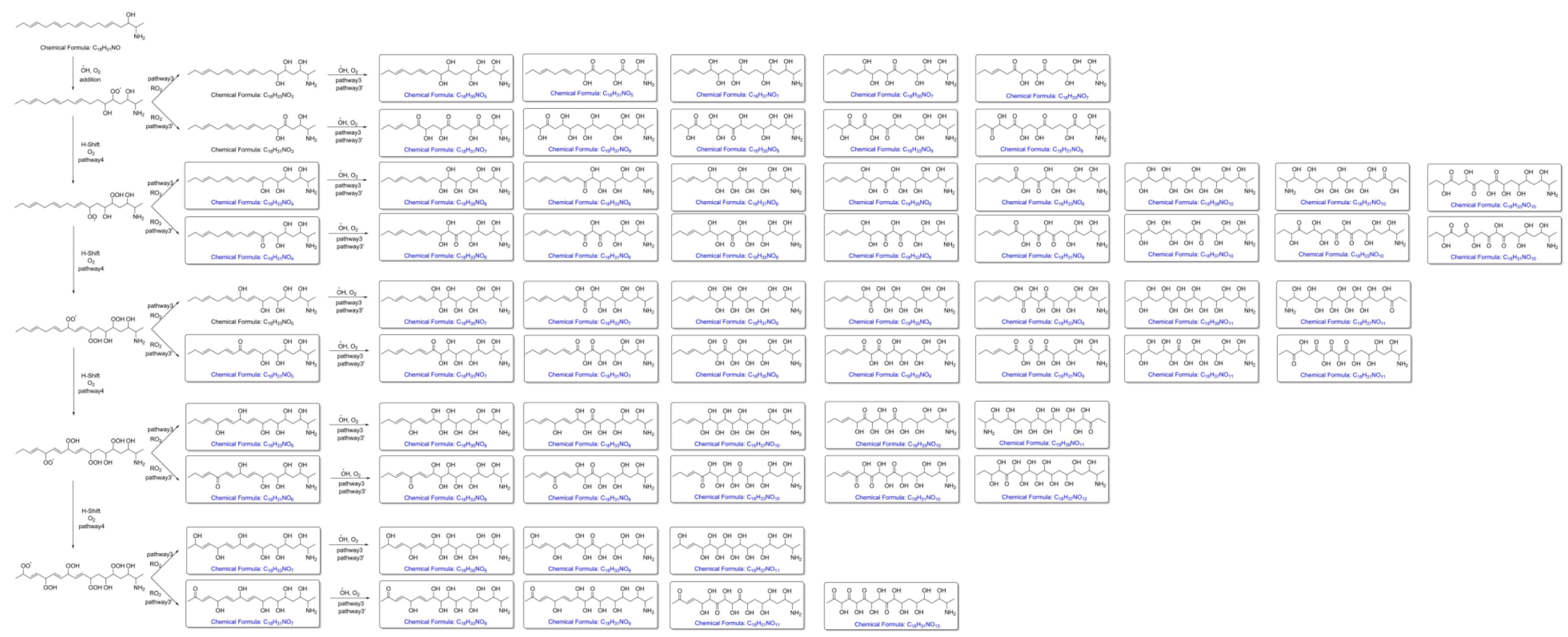

Figure S9. Simplified reaction scheme of the formation of oxygenated $\mathrm{CHON}$ compounds containing a $-\mathrm{NH}_{2}$ group from unsaturated $\mathrm{C}_{18}$ amino alcohol $\left(\mathrm{C}_{18} \mathrm{H}_{37} \mathrm{NO}_{4}\right)$ oxidation in the atmosphere. One representative structure is shown for each chemical formula. Chemical formulas in the boxes are found in the formula list detected in $10-18 \mathrm{~nm}$ particles. Pathway 3: $\mathrm{OH}$ and $\mathrm{O}_{2}$ addition followed by reaction with $\mathrm{RO}_{2}$ to form a $-\mathrm{OH}$ or a $-\mathrm{C}=\mathrm{O}$ group; Pathway 4: successive intermolecular $\mathrm{H}$-shift $/ \mathrm{O}_{2}$ addition autoxidation to form $\mathrm{RO}_{2}$ radicals with $-\mathrm{OOH}$ group. 
Table S1. Predicted saturation concentration $\left(\mathrm{C}^{*}\right)$ range of most abundant $\mathrm{CHON}$ and $\mathrm{CHO}$ formulas, as well as their possible precursors.

\begin{tabular}{|c|c|c|}
\hline Formula & Predicted $C^{*}\left(\mu \mathrm{g} \mathrm{m}^{-3}\right)$ & Predicted $\mathrm{C}^{*}$ of possible precursors $\left(\mu \mathrm{g} \mathrm{m}^{-3}\right)$ \\
\hline \multicolumn{3}{|c|}{ ESI- mode } \\
\hline $\mathrm{C}_{18} \mathrm{H}_{33} \mathrm{NO}_{4}$ & $1.62 \times 10^{-5}-2.06 \times 10^{-2}$ & $3.40 \times 10^{-1}-8.91$ \\
\hline $\mathrm{C}_{18} \mathrm{H}_{33} \mathrm{NO}_{6}$ & $7.66 \times 10^{-10}-1.33 \times 10^{-2}$ & $3.40 \times 10^{-1}-8.87 \times 10^{1}$ \\
\hline $\mathrm{C}_{18} \mathrm{H}_{34} \mathrm{~N}_{2} \mathrm{O}_{6}$ & $7.62 \times 10^{-11}-1.32 \times 10^{-3}$ & $3.40 \times 10^{-2}-8.91$ \\
\hline $\mathrm{C}_{18} \mathrm{H}_{34} \mathrm{~N}_{2} \mathrm{O}_{7}$ & $5.21 \times 10^{-13}-9.06 \times 10^{-6}$ & $3.40 \times 10^{-2}-8.91$ \\
\hline $\mathrm{C}_{18} \mathrm{H}_{34} \mathrm{~N}_{2} \mathrm{O}_{8}$ & $1.30 \times 10^{-15}-5.56 \times 10^{-6}$ & $3.40 \times 10^{-2}-8.91$ \\
\hline $\mathrm{C}_{18} \mathrm{H}_{36} \mathrm{~N}_{2} \mathrm{O}_{5}$ & $1.44 \times 10^{-8}-1.10 \times 10^{-2}$ & $3.40 \times 10^{-2}-8.87 \times 10^{1}$ \\
\hline $\mathrm{C}_{18} \mathrm{H}_{36} \mathrm{~N}_{2} \mathrm{O}_{6}$ & $9.83 \times 10^{-11}-7.54 \times 10^{-5}$ & $3.40 \times 10^{-2}-8.87 \times 10^{1}$ \\
\hline $\mathrm{C}_{18} \mathrm{H}_{36} \mathrm{~N}_{2} \mathrm{O}_{7}$ & $6.72 \times 10^{-13}-5.15 \times 10^{-7}$ & $3.40 \times 10^{-2}-8.87 \times 10^{1}$ \\
\hline $\mathrm{C}_{19} \mathrm{H}_{39} \mathrm{NO}_{7}$ & $3.40 \times 10^{-12}-1.15 \times 10^{-7}$ & $1.34 \times 10^{-1}-3.51 \times 10^{1}$ \\
\hline $\mathrm{C}_{30} \mathrm{H}_{57} \mathrm{NO}_{4}$ & $7.44 \times 10^{-8}-6.69 \times 10^{-6}$ & $4.29 \times 10^{-6}-1.16 \times 10^{-3}$ \\
\hline $\mathrm{C}_{30} \mathrm{H}_{59} \mathrm{NO}_{3}$ & $5.58 \times 10^{-9}-1.58 \times 10^{-6}$ & $4.29 \times 10^{-6}-1.14 \times 10^{-4}$ \\
\hline $\mathrm{C}_{30} \mathrm{H}_{59} \mathrm{NO}_{4}$ & $6.28 \times 10^{-10}-8.62 \times 10^{-6}$ & $4.29 \times 10^{-6}-1.16 \times 10^{-3}$ \\
\hline $\mathrm{C}_{30} \mathrm{H}_{59} \mathrm{NO}_{5}$ & $4.52 \times 10^{-12}-1.32 \times 10^{-6}$ & $4.29 \times 10^{-6}-1.16 \times 10^{-3}$ \\
\hline $\mathrm{C}_{30} \mathrm{H}_{59} \mathrm{NO}_{6}$ & $2.64 \times 10^{-13}-8.93 \times 10^{-9}$ & $4.29 \times 10^{-6}-1.16 \times 10^{-3}$ \\
\hline $\mathrm{C}_{30} \mathrm{H}_{60} \mathrm{O}_{6}$ & $1.66 \times 10^{-14}-3.76 \times 10^{-13}$ & $4.36 \times 10^{-5}-1.16 \times 10^{-3}$ \\
\hline $\mathrm{C}_{20} \mathrm{H}_{40} \mathrm{O}_{6}$ & $2.13 \times 10^{-10}-4.83 \times 10^{-9}$ & $5.28 \times 10^{-1}-1.39 \times 10^{1}$ \\
\hline $\mathrm{C}_{21} \mathrm{H}_{42} \mathrm{O}_{6}$ & $8.32 \times 10^{-11}-1.88 \times 10^{-9}$ & $2.07 \times 10^{-1}-5.46 \times 10^{0}$ \\
\hline $\mathrm{C}_{22} \mathrm{H}_{44} \mathrm{O}_{4}$ & $6.95 \times 10^{-7}-1.57 \times 10^{-5}$ & $8.15 \times 10^{-2}-2.15 \times 10^{0}$ \\
\hline $\mathrm{C}_{24} \mathrm{H}_{48} \mathrm{O}_{4}$ & $1.06 \times 10^{-7}-2.39 \times 10^{-6}$ & $1.25 \times 10^{-2}-3.3 \times 10^{-1}$ \\
\hline $\mathrm{C}_{26} \mathrm{H}_{52} \mathrm{O}_{4}$ & $1.60 \times 10^{-8}-3.63 \times 10^{-7}$ & $1.90 \times 10^{-3}-5.05 \times 10^{-2}$ \\
\hline $\mathrm{C}_{27} \mathrm{H}_{54} \mathrm{O}_{6}$ & $2.87 \times 10^{-13}-6.49 \times 10^{-12}$ & $7.42 \times 10^{-4}-1.97 \times 10^{-2}$ \\
\hline $\mathrm{C}_{28} \mathrm{H}_{56} \mathrm{O}_{4}$ & $2.41 \times 10^{-9}-5.47 \times 10^{-8}$ & $2.89 \times 10^{-4}-7.67 \times 10^{-3}$ \\
\hline $\mathrm{C}_{28} \mathrm{H}_{56} \mathrm{O}_{6}$ & $1.11 \times 10^{-13}-2.51 \times 10^{-12}$ & $2.89 \times 10^{-4}-7.67 \times 10^{-3}$ \\
\hline $\mathrm{C}_{29} \mathrm{H}_{58} \mathrm{O}_{6}$ & $4.29 \times 10^{-14}-9.73 \times 10^{-13}$ & $1.12 \times 10^{-4}-2.98 \times 10^{-3}$ \\
\hline $\mathrm{C}_{33} \mathrm{H}_{66} \mathrm{O}_{6}$ & $9.56 \times 10^{-16}-2.17 \times 10^{-14}$ & $2.54 \times 10^{-6}-6.77 \times 10^{-5}$ \\
\hline $\mathrm{C}_{38} \mathrm{H}_{76} \mathrm{O}_{8}$ & $3.66 \times 10^{-22}-8.30 \times 10^{-21}$ & $2.18 \times 10^{-8}-5.85 \times 10^{-7}$ \\
\hline \multicolumn{3}{|c|}{ ESI+ mode } \\
\hline $\mathrm{C}_{11} \mathrm{H}_{18} \mathrm{~N}_{4} \mathrm{O}_{8}$ & $4.73 \times 10^{-9}-3.63 \times 10^{-3}$ & $2.21 \times 10^{0}-5.61 \times 10^{2}$ \\
\hline $\mathrm{C}_{12} \mathrm{H}_{20} \mathrm{~N}_{4} \mathrm{O}_{8}$ & $1.85 \times 10^{-9}-1.42 \times 10^{-3}$ & $8.85 \times 10^{-1}-2.26 \times 10^{2}$ \\
\hline $\mathrm{C}_{19} \mathrm{H}_{35} \mathrm{NO}_{3}$ & $9.26 \times 10^{-4}$ & $1.34 \times 10^{-1}-2.23 \times 10^{-1}$ \\
\hline $\mathrm{C}_{19} \mathrm{H}_{36} \mathrm{~N}_{2} \mathrm{O}_{5}$ & $4.35 \times 10^{-9}-3.34 \times 10^{-3}$ & $1.34 \times 10^{-1}-3.52 \times 10^{0}$ \\
\hline $\mathrm{C}_{19} \mathrm{H}_{37} \mathrm{NO}_{3}$ & $1.20 \times 10^{-3}-2.71 \times 10^{-2}$ & $1.34 \times 10^{-1}-2.23 \times 10^{-1}$ \\
\hline
\end{tabular}




\begin{tabular}{lcl}
\hline $\mathrm{C}_{19} \mathrm{H}_{38} \mathrm{~N}_{2} \mathrm{O}_{3}$ & $2.71 \times 10^{-3}-5.04 \times 10^{-3}$ & $1.34 \times 10^{-2}-3.52 \times 10^{0}$ \\
$\mathrm{C}_{24} \mathrm{H}_{46} \mathrm{~N}_{2} \mathrm{O}_{4}$ & $5.73 \times 10^{-9}-4.39 \times 10^{-3}$ & $1.24 \times 10^{-4}-3.28 \times 10^{-2}$ \\
$\mathrm{C}_{25} \mathrm{H}_{43} \mathrm{NO}_{4}$ & $3.07 \times 10^{-7}-1.72 \times 10^{-5}$ & $4.83 \times 10^{-4}-1.28 \times 10^{-2}$ \\
$\mathrm{C}_{26} \mathrm{H}_{51} \mathrm{NO}_{5}$ & $1.73 \times 10^{-9}$ & $1.88 \times 10^{-4}-3.13 \times 10^{-4}$ \\
$\mathrm{C}_{27} \mathrm{H}_{50} \mathrm{~N}_{2} \mathrm{O}_{4}$ & $6.42 \times 10^{-10}-3.29 \times 10^{-7}$ & $7.23 \times 10^{-6}-1.95 \times 10^{-3}$ \\
$\mathrm{C}_{27} \mathrm{H}_{50} \mathrm{~N}_{2} \mathrm{O}_{5}$ & $3.11 \times 10^{-11}-1.05 \times 10^{-6}$ & $7.23 \times 10^{-6}-1.95 \times 10^{-3}$ \\
$\mathrm{C}_{27} \mathrm{H}_{52} \mathrm{~N}_{2} \mathrm{O}_{3}$ & $4.94 \times 10^{-8}-2.76 \times 10^{-6}$ & $7.23 \times 10^{-6}-1.95 \times 10^{-3}$ \\
$\mathrm{C}_{28} \mathrm{H}_{52} \mathrm{~N}_{2} \mathrm{O}_{6}$ & $1.14 \times 10^{-14}-8.03 \times 10^{-8}$ & $2.81 \times 10^{-6}-7.57 \times 10^{-4}$ \\
$\mathrm{C}_{28} \mathrm{H}_{54} \mathrm{~N}_{2} \mathrm{O}_{6}$ & $5.95 \times 10^{-15}-1.03 \times 10^{-7}$ & $2.81 \times 10^{-6}-7.57 \times 10^{-4}$ \\
$\mathrm{C}_{28} \mathrm{H}_{56} \mathrm{~N}_{2} \mathrm{O}_{3}$ & $6.09 \times 10^{-8}-1.38 \times 10^{-6}$ & $2.81 \times 10^{-6}-7.57 \times 10^{-4}$ \\
$\mathrm{C}_{28} \mathrm{H}_{56} \mathrm{~N}_{2} \mathrm{O}_{6}$ & $1.89 \times 10^{-14}-5.88 \times 10^{-9}$ & $2.81 \times 10^{-6}-7.57 \times 10^{-4}$ \\
$\mathrm{C}_{28} \mathrm{H}_{58} \mathrm{~N}_{2} \mathrm{O}_{3}$ & $3.18 \times 10^{-8}$ & $2.81 \times 10^{-6}-4.66 \times 10^{-6}$ \\
$\mathrm{C}_{29} \mathrm{H}_{56} \mathrm{~N}_{2} \mathrm{O}_{6}$ & $2.30 \times 10^{-15}-3.99 \times 10^{-8}$ & $1.09 \times 10^{-6}-2.94 \times 10^{-4}$ \\
$\mathrm{C}_{29} \mathrm{H}_{59} \mathrm{NO}_{7}$ & $2.85 \times 10^{-17}-6.47 \times 10^{-16}$ & $1.11 \times 10^{-5}-2.94 \times 10^{-4}$ \\
$\mathrm{C}_{33} \mathrm{H}_{59} \mathrm{NO}_{5}$ & $1.05 \times 10^{-12}-3.02 \times 10^{-8}$ & $2.49 \times 10^{-7}-6.65 \times 10^{-6}$ \\
$\mathrm{C}_{34} \mathrm{H}_{59} \mathrm{NO}_{6}$ & $2.13 \times 10^{-15}-1.38 \times 10^{-9}$ & $9.64 \times 10^{-8}-2.57 \times 10^{-6}$ \\
$\mathrm{C}_{34} \mathrm{H}_{66} \mathrm{~N}_{2} \mathrm{O}_{3}$ & $1.15 \times 10^{-11}-3.58 \times 10^{-9}$ & $9.45 \times 10^{-9}-2.57 \times 10^{-6}$ \\
$\mathrm{C}_{34} \mathrm{H}_{68} \mathrm{~N}_{2} \mathrm{O}_{3}$ & $2.03 \times 10^{-10}-4.61 \times 10^{-9}$ & $9.45 \times 10^{-9}-2.57 \times 10^{-6}$ \\
$\mathrm{C}_{34} \mathrm{H}_{68} \mathrm{~N}_{2} \mathrm{O}_{5}$ & $3.75 \times 10^{-15}-2.88 \times 10^{-9}$ & $9.45 \times 10^{-9}-2.57 \times 10^{-6}$ \\
\hline
\end{tabular}

